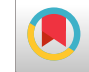

\title{
Post-Treatment Neurocognitive Disorders in Adults with Frontal or Parietal Astrocytomas
}

\author{
Kazem Anvari, ${ }^{1}$ Roham Salek, ${ }^{1,}$ Azar Fanipakdel, ${ }^{1}$ Roya Parsamanesh, ${ }^{2}$ Seyyed Mojtaba Ejlalzadeh, ${ }^{2}$ \\ Nafise Rahimy, ${ }^{3}$ and Seyed Alireza Javadinia ${ }^{4}$ \\ ${ }^{1}$ Cancer Research Center, Faculty of Medicine, Mashhad University of Medical Sciences, Mashhad, Iran \\ ${ }^{2}$ Faculty of Medicine, Islamic Azad University, Mashhad, Iran \\ ${ }^{3}$ Faculty of Nursing and Midwifery, Birjand University of Medical Sciences, Birjand, Iran \\ ${ }^{4}$ Student Research Committee, Faculty of Medicine, Mashhad University of Medical Sciences, Mashhad, Iran \\ "Corresponding author: Roham Salek,Cancer Research Center, Faculty of Medicine, Mashhad University of Medical Sciences, Mashhad, Iran. Tel: +98-5138426936, E-mail: \\ salekr@mums.ac.ir
}

Received 2016 January 28; Accepted 2016 March 15.

\begin{abstract}
Background: High-grade astrocytomas are among the most common neuroepithelial brain tumors. Because of their highly malignant nature, in most cases, in addition to maximal resection, they also require adjuvant treatments, such as chemotherapy and radiotherapy. A common accompanying condition, which may occur with this type of clinical presentation, is cognitive disorders. These can be caused by the tumor itself, the treatment used or may be patient related. The aim of this study was to evaluate the level of cognitive ability in patients with astrocytoma compared to the normal population and evaluating the factors possibly affecting them.

Methods: A case-control study was performed on 30 adults referred to Imam Reza and Omid hospitals, Mashhad, Iran, during year 2014. The studied patients had astrocytomas, for which they had performed surgery. All patients had also received radiotherapy. The control group consisted of 30 healthy individuals, among the patients' family members, who were matched for age and gender with the patients. The tools used in this study were a checklist for demographic data, and the Farsi version of Addenbrook's cognitive questionnaire. Data were entered in the SPSS 22 software and analyzed using the Student's t test and Mann-Whitney test. P values of $\leq 0.05$ were considered significant.

Results: Normal cognitive disorders were seen in 33.3\% and 80\% of the patient and control groups, respectively. Mild cognitive disability was observed in $10 \%$ of both groups; and Alzheimer's was observed in $56.7 \%$ and $10 \%$ of the patient and control groups, respectively. A statistically significant difference was found between cognitive function, age, and gender $(\mathrm{P}=0.0001$ in both $)$. No meaningful difference, however, was observed between cognitive score and tumor location, chemotherapy, and the time, from which treatment had ended.

Conclusion: With the high prevalence of cognitive disorders among patients with astrocytoma, one can conclude that the tumor itself and the surrounding factors affect the cognitive function of the patient. Results of this study showed that the type of treatment and some properties of the tumor, such as the tumor's location, do not affect the patient's cognitive capacity.
\end{abstract}

Keywords: Cognitive Disorder, Astrocytoma, Frontal Lobe, Temporal Lobe

\section{Background}

Astrocytomas are a group of brain tumors originating from glial cells, which are among the most common adult neoplasms (1). Based on the degree of malignancy, severity of symptoms, and amount of residual disease after surgery, the treatment may vary from simple resection to resection with chemoradiotherapy together with adjuvant chemotherapy. Neurocognitive disorders in adults with brain tumors are associated with type of disease, choice of treatment, and the patient's general condition (primary level of function).
Regarding the disease type, it has been shown that tumors with a higher degree of malignancy (grades 3 or 4 ) cause more neurocognitive dysfunction compared to low grade tumors (2). Some researchers believe otherwise, and state that neurocognitive disorders have nothing to do with the grade of tumor and are only related to treatment options, as well as tumor location (3). Patient-related factors, which may affect neurocognitive problems, include age, psychological state (in particular the level of depression) associated with the disease, and existence of comorbidities, and the state of performance $(4,5)$.

Different opinions have been stated about the role of 
different brain tumor treatments on neurocognitive function. In radiotherapy patients, neurocognitive impairment can be affected by total dose, amount of treatment, and fraction dose (6-8). Most studies in this field have focused on children with meduloblastomas (9) or leukemia (10), who had received whole brain radiotherapy. Less data exists regarding partial brain irradiation and neurocognitive problems in both adults and children. The majority of studies about neuro-cognitive disorder caused by brain tumors in adults have limitations, such as small sample size, (11-13), use of non-specific or non-sensitive tools (14, 15 ) or high heterogeneity of the sample population (regarding histology of the tumor and its location) $(16,17)$. In a study on 245 patients, it was shown that the effect of having surgery on neurocognitive function was considerably higher than its relation with the grading of the tumor (3). In another study, which was performed in 2001, results indicated that in patients with brain tumor undergoing radiotherapy, neurocognitive impairment was mostly related to having surgery (18). Nevertheless, studies evaluating the relative danger of various treatment modalities in causing neurocognitive disorders have rarely been performed (19). The potential of low grade tumors causing neurocognitive impairment is important, considering their long survival time and low age of onset (20). On the other hand, many issues accompanying high grade gliomas, such as brain edema, neurologic symptoms, and psychologic symptoms are all associated with neurocognitive dysfunction. Furthermore, some reports have even shown that before commencing treatment, up to $80 \%$ of such patients have memory dysfunction as well as executive function deficits (21).

Among the various brain tumor-related causes of neurocognitive disorders (including factors related to the tumor, patient, and treatment), it seems that only treatmentrelated factors can be modified in order to control these disorders. Three-dimensional conformal radiotherapy uses CT scan (or recently magnetic resonance imaging (MRI) fusion) in order to reduce the total dose up to $50 \%$ and reduce the high dose radiation of normal brain tissue up to $30 \%$ (22). In the long run, this reduction in normal brain tissue irradiation could lead to reduction in neurocognitive problems (23).

Considering the importance of the occurrence of these cognitive disorders caused by the various causes pertaining to brain tumors, this study was performed in order to assess the cognitive function of patients with astrocytomas and compare them with a healthy population, while studying factors, which influence cognitive function.

\section{Methods}

This case-control study was performed at the radiology and oncology wards of Omid and Imam Reza hospitals in Mashhad, during year 2014. These 2 hospitals were teaching hospitals of Mashhad University of Medical Sciences. For the study group, 30 patients with frontal or temporal lobe astrocytoma were entered in the study in a nonrandom consecutive manner, as they referred to the 2 hospitals. After being matched for age and gender, the control group were selected from the relatives of the study group. Criteria for entering the study were age between 16 and 75 years, the ability to completely speak and understand the Farsi language, completion of the standard treatment process, including brain surgery and radiotherapy with or without adjuvant chemotherapy, and having brain imaging performed at least once in the past 6 months. Exclusion criteria for both groups were history of a clear psychological illness, having another malignancy in addition to brain astrocytoma, having a known medical illness, history of more than one surgery or course of radiotherapy, remittance or progression of disease (increased size of tumor or amount of edema), a pathology showing mixed glioma or a non-astrocytoma component, and taking medications, which affect cognitive function.

The Addenbrook's cognitive examination (ACE) questionnaire was used for data collection. This questionnaire had 5 fields, each evaluating a different cognitive function. The highest score for this test was 100, which is made up of the following: attention and directions (18 points), memory (26 points), speech fluency (14 points), language (26 points), and visuospatial abilities (16). A higher score reflects better function in the respective field. The form is completed by the researcher or research units. In Iran, the validity of the Farsi version of the ACE questionnaire was proven in the study of Pouretemad. Sensitivity and specificity of ACE in alzheimer's disease (AD) and mild cognitive impairment (MCI) was evaluated. At the 84 cut-off point, the Farsi version of ACE had a sensitivity of $93 \%$ and specificity of $91 \%$ in differentiating $\mathrm{MCI}$ from the normal population. At the cut of point of 78 , it also had a sensitivity of $73 \%$ and specificity of $93 \%$ in differentiating AD from MCI; and at a similar cut off point, had 100\% sensitivity and 96\% specificity in differentiating AD from the normal population. A score of 85 or more was considered normal. A score between 78 and 85 showed mild cognitive impairment, and less than 78 was indicative of AD. The calculated Cronbach's alpha in this study was 0.84 overall (24).

Data collection was performed by interviewing patients. While visiting the patients, their companions were also asked to participate in the interview.

After collection, the data was entered in the SPSS 22 and 
analyzed using Kruskal-Wallis, Mann-Whitney, and Shapiro Wilk tests, with a level of significance of $\mathrm{P} \leq 0.05 \%$.

\section{Results}

In total, 30 people were entered in the case group and 30 people in the control group. The participants were age and gender matched. The demographic data of both groups are shown in Table1. Regarding the type of tumor in the patient group, 50\% had a grade IV tumor, of which $60 \%$ had a frontal lobe tumor. Radiotherapy was performed on all patients, while 70\% had received chemotherapy. Most patients had finished radiotherapy 6 months or more before entering the study (56.7\%) (Table 2).

Comparison of the number of cognitive disabilities in patients with frontal or parietal astrocytoma between groups showed that the rate of MCI was 10\% in both groups, while Alzheimer's disease was $56.7 \%$ and $10 \%$ in the patient and control group, respectively (Table 3 ).

When comparing cognitive disorders between the groups based on age, it was shown that cognitive function was significantly higher in the control group (0.001). This difference was also observed in all the sub-categories of the cognitive exam, including attention and direction (0.0001), speech fluency (0.001), language (0.014), and visuospatial abilities (0.002). The results also showed that in males, the average of attention and direction (0.0001), memory (0.003), speech fluency (0.0003), and visuospatial ability (0.002) was significantly higher in the control group compared to the patient group. This was while no such difference was observed among females between the 2 groups (Table 4 ).

Comparison of tumor location (frontal and parietal), performing chemotherapy, and time of ending radiotherapy showed no meaningful difference (Table 5).

\section{Discussion}

In this study, prevalence of cognitive disorders, with some influential factors, was evaluated in patients with frontal or parietal astrocytoma. After studying the patient documents and extracting the data related to the tumor, both the demographic as well as Addenbrook's questionnaire were completed by both the study and control groups (which were companions of patients in the hospital, who did not have criteria for exclusion from the study and were matched for age and gender with the patient group), and the relationship between the results of the frequency of cognitive impairment and underlying factors and tumor-related factors were investigated.
Thirty patients with astrocytoma that were under follow-up after their treatment and 30 people as the control group participated in this study. The results revealed that in the patient group with astrocytoma, 56.7\% had cognitive impairment at the level of Alzheimer's classification and 33.3\% of the patients were in the normal group (without cognitive impairment), and $10 \%$ of them in the mild cognitive impairment group. In contrast, in the control group, $80 \%$ of the subjects were in the normal range and in the normal group, $10 \%$ in the mild cognitive impairment group, and 10\% in the Alzheimer's group. These results indicate that cognitive impairment in patients was clearly higher than that of the control group.

These results are in line with previous studies; for example in the study of Habets et al. (25) (2014) conducted in the Netherlands that was performed to assess the impact of tumor and surgery on cognitive function in patients with high-grade glioma, the cognitive performance score in patients had significantly declined compared with the control group $(\mathrm{P}<0.001)$.

Similarly, in one study conducted by Zarghi et al. (2011) (26) in Tehran on the effect of surgery on psychological disorders in patients with brain glioma, the mean score of the MMSE questionnaire before surgery was $22.2 \%$, and after that was 10 , which clearly implied that the cognitive function of patients would be reduced after surgery. $(\mathrm{P}=0.00)$

In other studies, due to lack of utilization of a common test, the overall score for cognitive function was not mentioned; yet the scores of each area and how they change was stated. In a study by Correa et al. (27) (2008) in New York, which was performed with the aim of pursuing cognitive function in patients with low-grade glioma, at the initiation of the evaluation in patients, who were cured, in the extent of performance and movement speed, significant cognitive impairment was evident.

In a research study by Bosma et al. (28) (2006) from the Netherlands, which examined cognitive processes in patients with glioma and in particular the effects of tumor recurrence in these patients; the cognitive function of patients in the areas of psychomotor activity $(\mathrm{P}=0.017)$, information processing $(\mathrm{P}=0.041)$, and attention $(\mathrm{P}=0.030)$ were reduced significantly. This study showed a significant reduction of cognitive function in patients with high grade glioma.

In another study performed by Dr. Talacchi et al. (2010) in Italy, they investigated the cognitive effects of tumor and surgical treatment in patients with glioma, and the result showed that the cognitive function of patients in the areas of attention and memory was significantly reduced after surgery $(\mathrm{P}<0.05)$.

In the present study, $43 \%$ of patients and the control group were less than 37 years old, and $57 \%$ of them were 


\begin{tabular}{|c|c|c|c|c|}
\hline \multirow[t]{2}{*}{ Variable } & \multicolumn{2}{|c|}{ Study Group } & \multicolumn{2}{|c|}{ Control Group } \\
\hline & Frequency & $\%$ & Frequency & $\%$ \\
\hline \multicolumn{5}{|l|}{ Education } \\
\hline Less than diploma & 20 & 65.5 & 14 & 55 \\
\hline Diploma and less than Bachelor's & 6 & 20.7 & 3 & 5 \\
\hline Bachelor's & 4 & 13.8 & 10 & 35 \\
\hline Master's & 0 & 0 & 3 & 5 \\
\hline \multicolumn{5}{|l|}{ Gender } \\
\hline Male & 24 & 80 & 22 & 73.3 \\
\hline Female & 6 & 20 & 8 & 26.7 \\
\hline \multicolumn{5}{|l|}{ Age } \\
\hline$<37$ & 13 & 43.3 & 13 & 43.3 \\
\hline 37 or more & 17 & 56.7 & 17 & 56.7 \\
\hline
\end{tabular}

Table 2. Data Regarding Patients' Tumors

\begin{tabular}{|ccc}
\hline Variable & Frequency & $\%$ \\
\hline Tumor grade & 0 & 0 \\
\hline Grade I & 10 & 33.3 \\
\hline Grade II & 5 & 15 \\
\hline Grade III & 15 & 50 \\
\hline Grade IV & 18 & 60 \\
\hline Tumor location & 12 & 40 \\
\hline Frontal & & 30 \\
\hline Parietal & 9 & 70 \\
\hline Chemotherapy & 21 & \\
\hline No & 17 & 43.3 \\
\hline Yes & & 56.7 \\
\hline End time of radiotherapy & 13 & \\
\hline Less than 6 months & & \\
\hline 6 months and more & & \\
\hline
\end{tabular}

over 37 years old. Overall, $8 \%$ of patients, who were under 37 years old, were in the mild cognitive insufficiency group, $46 \%$ in the normal group, and $46 \%$ in the Alzheimer's group. All those in the control group under the age of 37 were normal. Furthermore, $12 \%$ of patients, who were 37 years and older, were categorized in the group with mild cognitive impairment, $24 \%$ in the normal group, and $64 \%$ in the Alzheimer's group. Seventeen percent of the control group, who were 37 years and older, were in the group of mild cognitive impairment, $17 \%$ in the Alzheimer's group, and $66 \%$ in the normal group. Comparing cognitive function between control and patient groups, who were less than 37 years old, revealed a significant difference between them. This condition was observed in all areas of cognitive performance test except memory. Therefore, it could be concluded that cognitive function in the control group is significantly higher than the patient group $(\mathrm{P}=0.001)$. The mean of attention and direction $(\mathrm{P}=0.0001)$, verbal fluidity $(\mathrm{P}=0.001)$, language $(\mathrm{P}=0.014)$, and visuospatial capabilities $(\mathrm{P}=0.002)$ in the control group was significantly higher. In the case of the group of 37 years and older, the cognitive function of the control group was significantly higher than the patients $(\mathrm{P}=0.0001)$. Therefore, it could be concluded that the factor of age plays a role in the patients' cognitive functions.

In the study performed by Bosma et al. (28), which aimed at examining cognitive function in patients with glioma, as age increased, the level of cognitive performance decreased $(\mathrm{P}=0.00)$.

In addition, in the study of Habets et al. (25), there was a significant correlation between age and cognitive function in patients after surgery.

In contrast to the current study, the study done by Dr. Correa et al. (29) (2007) in New York in order to evaluate cognitive function in patients with low-grade Glioma and the effects of disease and treatment, the results showed no significant relationship between the 2 groups of patients with glioma in relation to their age. The mean age in 40 patients participating in this study was 41.5 years, which was different from the mean age (37 years).

In the present study, 24 patients were males and 6 were females. The control group was almost identical 
Table 3. Cognitive Impairment in patients with Frontal or Temporal Asterocytomas

\begin{tabular}{|c|c|c|c|c|c|}
\hline \multirow[t]{2}{*}{ Variable } & \multirow[t]{2}{*}{ Sub-Group } & \multicolumn{2}{|c|}{ Patient Group } & \multicolumn{2}{|c|}{ Control Group } \\
\hline & & Frequency & $\%$ & Frequency & $\%$ \\
\hline \multirow{3}{*}{ Cognitive function } & Normal & 10 & 33.3 & 24 & 80 \\
\hline & Mild cognitive impairment & 3 & 10 & 3 & 10 \\
\hline & Alzheimer's & 17 & 56.7 & 3 & 10 \\
\hline
\end{tabular}

Table 4. Comparison of Addenbrook's Cognitive Examination-R score in Patient and Control Groups Based on Age and Gender ${ }^{\mathrm{a}}$

\begin{tabular}{|c|c|c|c|c|}
\hline Variables & Control Group & Patient Group & Mann-Whitney & Value \\
\hline \multicolumn{5}{|l|}{ Age } \\
\hline$<37$ & $95.15 \pm 2.609$ & $80.92 \pm 11.765$ & 24.5 & $0.001^{* *}$ \\
\hline$\geq 37$ & $86.12 \pm 7.507$ & $58.59 \pm 25.222$ & 46.5 & $0.0001^{* *}$ \\
\hline \multicolumn{5}{|l|}{ Gender } \\
\hline Female & $90.25 \pm 6.606$ & $76.83 \pm 13.776$ & 9 & 0.059 \\
\hline
\end{tabular}

${ }^{\mathrm{a}}$ Values are expressed as mean $\pm \mathrm{SD}$.

Table 5. Comparison of Patients' Cognitive Function According to Tumor Location, Chemotherapy, and Time of Ending Radiotherapy

\begin{tabular}{|c|c|c|c|c|c|c|}
\hline \multirow[t]{2}{*}{ Cognitive Function } & \multicolumn{2}{|c|}{ Frontal } & \multicolumn{2}{|c|}{ Parietal } & \multirow[t]{2}{*}{ Mann-Whitney } & \multirow[t]{2}{*}{ Value } \\
\hline & Mean & SD & Mean & SD & & \\
\hline ACE-R & 73.06 & 20.054 & 61.08 & 26.363 & 79 & 0.232 \\
\hline \multirow[t]{2}{*}{ Cognitive function } & \multicolumn{2}{|c|}{ No chemotherapy } & \multicolumn{2}{|c|}{ Chemotherapy } & Mann-Whitney & value \\
\hline & Mean & SD & Mean & SD & & \\
\hline \multirow[t]{2}{*}{ Cognitive function } & \multicolumn{2}{|c|}{ Less than 6 months } & \multicolumn{2}{|c|}{6 months and more } & Mann-Whitney & value \\
\hline & Mean & SD & Mean & SD & & \\
\hline ACE-R & 61.69 & 27.299 & 73.29 & 18.674 & 86.5 & 0.320 \\
\hline
\end{tabular}

with the patient group. The level of cognitive function in the male patients was $8.3 \%$ in the group of mild cognitive insufficiency, 33.3\% in the normal group, and 58.3\% in the Alzheimer's group. Considering results of measuring cognitive function among the female patient group, $16.7 \%$ were in the mild insufficiency group, 33.3\% were female in the normal group, and 50\% in the Alzheimer's group. In the control group of males, $4.5 \%$ were in the mild cognitive insufficiency group, $13.6 \%$ in the Alzheimer's group, and $81.8 \%$ in the normal group. Overall, $25 \%$ of females in the control group were in the group of mild cognitive impairment and 75\% in the normal group. Mean score of cognitive function among males of the control group was significantly higher than male patients $(\mathrm{P}=0.0001)$. The overall score of cognitive function based on the ACR questionnaire in males of the control group was 89 versus 60 in the patient group. Also, in the male group, the average rate of attention and orientation $(\mathrm{P}=0.0001)$, memory $(\mathrm{P}=0.003)$, verbal fluidity $(\mathrm{P}=0.0001)$, language $(\mathrm{P}=0.003)$, and visuospatial abilities $(\mathrm{P}=0.0001)$ of the control group were significantly higher than the patient group. However, in case of the female group, there were no significant differences between the 2 control and patient groups $(\mathrm{P}=0.059)$. Based on these results, one can infer that there is a significant relationship between cognitive performance score and gender.

However, unlike the current study, in Habets et al.'s study (25), patients, who were unable to fully complete cognitive testing were clearly older and most of them were female $(P=0.009)$. There is significant evidence suggesting 
that females have more cognitive impairment than males. This difference in the results of these studies could be due to differences in the number of females and males, who had participated in both studies (24 males versus 6 females in the current study compared with 9 males versus $14 \mathrm{fe}$ males in the Ester study).

The result of another study that was performed by Bosma et al. (28) showed that there was no significant relationship between gender and cognitive function of patients $(\mathrm{P}=0.714)$.

The result of another study conducted by Laack et al. (2005) in the United States, did not reveal a significant relationship between gender and cognitive function among patients with low grade glioma after radiotherapy $(\mathrm{P}>$ 0.05).

In the current study, the authors evaluated the frequency of cognitive impairment in patients with astrocytoma based on tumor location. In general, $5.6 \%$ of the patients with frontal astrocytoma were in the group with mild cognitive insufficiency, $44.4 \%$ in the normal group, and $50 \%$ in the Alzheimer's group. Furthermore, 16.7\% of the patients with parietal astrocytoma were in the normal group, $16.7 \%$ in the mild impairment group, and $66.7 \%$ in the Alzheimer's group. However, there was no significant difference between mean cognitive impairment among patients with frontal area astrocytoma and the rate of cognitive impairment in patients with parietal astrocytoma ( $P$ $=0.0232$ ). However, in general, the mean score of cognitive function in patients with frontal tumors was higher than patients with parietal astrocytoma.

These results are in line with the results of Bosma et al. (28) (2007) from the Netherlands that examined cognitive processes in patients with glioma and in particular those, who had tumor recurrence in this study, also, no significant relationship was found between the cognitive function score and the location of the tumor $(\mathrm{P}=0.270)$.

In the study of Dr. Habets et al. (25) (2014), which was conducted in the Netherlands to investigate the effect of tumor and surgery on cognitive function in patients with high grade glioma, no significant relationship was found between tumor location and cognitive function of patients.

In the study of Laack et al. (30) from the United States, cognitive function after radiotherapy in patients with low grade glioma was investigated. There was no significant relationship between tumor location and cognitive function $(\mathrm{P}>0.05)$.

In the present study, $9.5 \%$ of patients under chemotherapy treatment were in the mild cognitive insufficiency group, 33.3\% in the normal group, and $57.1 \%$ in the Alzheimer's group. Furthermore, 11.1\% of patients, who had not undergone chemotherapy treatment, were in the group with mild cognitive impairment, 33.3\% in the normal group, and 55.6\% in the Alzheimer's group. The overall score of cognitive function of patients, who had not undergone chemotherapy treatment based on the ACR was 76 , and the overall score of patients, who had undergone chemotherapy treatment was 64 . However, regarding cognitive impairment among patients, who had undergone chemotherapy treatment and those, who did not use this type of treatment, there was no significant difference $(\mathrm{P}=$ 0.397).

It should be noted that although a meaningful relationship with chemotherapy was not found, in the group that did not undergo chemotherapy treatment, the performance score in all 5 cognitive domains was higher than those, who took chemotherapy treatment. For example, in the memory domain, the mean ACR score in patients, who did not take chemotherapy was 16 compared with the score of 14 for patients, who had undergone chemotherapy treatment.

These results are consistent with the study of Prabhu et al. (31) (2014) in the United States that investigated the effect of chemotherapy and radiotherapy on cognitive function of patients with low grade glioma. In this study, after follow-up radiotherapy with or without chemotherapy, no significant relationship was found between the 2 groups in terms of cognitive function score $(\mathrm{P}=0.99)$.

In a study performed by Correa et al. (29) (2006) in New York, they evaluated the cognitive performance of patients with low-grade glioma and the effects of disease and its treatment revealed that, although, the score of the questionnaire of verbal memory and non-verbal memory was lower in the group, who had not undergone chemotherapy treatment than those, who had undergone chemotherapy treatment, yet there was no significant difference between the 2 groups $(\mathrm{P}>0.05)$.

To determine the frequency of cognitive impairment in terms of tumor grade, the results of this study showed that $10 \%$ of patients with grade II were in the group of mild cognitive impairment, $40 \%$ were in the normal group, and $50 \%$ were in the Alzheimer's group. Twenty percent of patients with grade III, were in the group of mild cognitive insufficiency, $40 \%$ in the normal group, and $40 \%$ in the Alzheimer's group. Furthermore, 6.7\% of the patients with grade IV were in the group with mild cognitive insufficiency, 26.7\% in the normal group, and $66.7 \%$ in the Alzheimer's group. The results indicate that there was no significant difference between the mean of cognitive impairment of astrocytoma patients in different tumor grades $(P=0.306)$. Therefore, it could be said that the type of grade did not affect the level of cognitive impairment of patients. Of course, it should be noted that although no significant relationship between cognitive 
function and degree of tissue differentiation was observed, yet at the highest degree of tissue differentiation (grade 4), the largest amount of cognitive impairment was observed (Alzheimer's group: 66.7\%). By increasing the degree of histological differentiation, the questionnaire score and cognitive function of these patients decreased. This finding could be due to the volume of radiotherapy field and the higher dose of radiotherapy used for these patients. However, despite the presence of more Alzheimer's patients in this group, there is no significant difference, which is probably due to the smaller sample size of the current study.

In contrast to this study, Talachi et al. (2010) found a significant relationship with the degree of tumor differentiation and higher cognitive impairment was observed with increased tumor grade $(P=0.001)$.

In the study of Bosma et al. (28), a significant relationship was found between tumor grade and cognitive impairment $(\mathrm{P}=0.000)$. This difference could be due to the groups of patients. In the study of Bosma et al. (28), the patients had only high grade gliomas. However, the current study included all patients with glioma, both low and high grade. However, in the study of Bosma et al. (28), the majority of patients with low cognitive performance score had grade 4 tumors, which is line with the current study.

The last variable evaluated in this study was to determine the frequency of cognitive impairment in patients with astrocytoma, according to the time of completion of radiotherapy. The results showed that $7.7 \%$ of patients with radiotherapy less than 6 months had a mild deficiency, $23.1 \%$ were in the normal range, and $69.2 \%$ were in the Alzheimer's range. Also, $11.8 \%$ of the patients with 6 months or more of radiotherapy were in the range of mild insufficiency, $41.2 \%$ were in the normal group, and $47.1 \%$ were in the Alzheimer's group. There was no significant difference between mean cognitive impairment of astrocytoma patients, whose radiotherapy time was less than 6 months and the mean of patients, whose radiotherapy time was 6 months or more. Therefore, one can conclude that the duration of radiotherapy did not have a significant effect on the degree of cognitive impairment in patients ( $P$ $=0.320$ ), and in all cognitive areas, the performance score in the group that had completed less than 6 months of treatment was lower than the other group.

In a study by Laack et al. (30) in the United States, which examined cognitive function after radiotherapy in patients with low grade glioma, despite a slight increase in cognitive function of patients, there was a follow up of 12 months and 60 months after radiotherapy, yet no significant relationship was found.

\subsection{Conclusion}

The rate of cognitive impairment in the patient group was $66.7 \%$, which indicates a high incidence of cognitive impairment in patients with cerebral astrocytoma. In addition, a significant relationship was found between age and cognitive function, which could lead to the conclusion that as age increases, the incidence of cognitive impairment also increases. On the other hand, males showed more cognitive impairment than females. However, no significant relationship was found between cognitive function and prognostic factors of cancer, including the degree of histologic differentiation and position of the tumor and treatment factors, such as chemotherapy or the duration of treatment. However, due to the prevalence of cognitive impairment in patients with astrocytoma and the effect of these impairments on the quality of life of the individual, it seems important for these patients to be assessed for cognitive impairment before treatment and in different stages of treatment. If cognitive impairment is found in these patients, measures, such as educational and supportive psychotherapy could be taken in which the nature and course of their illness will clearly be described and at the same time they will be able to accept their disabilities. Furthermore, by identifying and enhancing healthy functional areas as well as using appropriate medical therapy, the quality of life of an individual could be improved.

\section{References}

1. Barnholtz-Sloan JS, Sloan AE, Schwartz AG. Relative survival rates and patterns of diagnosis analyzed by time period for individuals with primary malignant brain tumor, 1973-1997. J Neurosurg. 2003;99(3):458-66. doi: 10.3171/jns.2003.99.3.0458. [PubMed: 12959430].

2. Hom J, Reitan RM. Neuropsychological correlates of rapidly vs. slowly growing intrinsic cerebral neoplasms. J Clin Neuropsychol. 1984;6(3):309-24. doi: 10.1080/01688638408401221. [PubMed: 6470167].

3. Scheibel RS, Meyers CA, Levin VA. Cognitive dysfunction following surgery for intracerebral glioma: influence of histopathology, lesion location, and treatment. J Neurooncol. 1996;30(1):61-9. doi: 10.1007/BF00177444. [PubMed: 8865004].

4. Grant R, Slattery J, Gregor A, Whittle IR. Recording neurological impairment in clinical trials of glioma. J Neurooncol. 1994;19(1):37-49. doi: 10.1007/BF01051047. [PubMed: 7815103].

5. Murray KJ, Nelson DF, Scott C, Fischbach AJ, Porter A, Farnan N, et al. Quality-adjusted survival analysis of malignant glioma. Patients treated with twice-daily radiation (RT) and carmustine: a report of Radiation Therapy Oncology Group (RTOG) 83-02. Int J Radiat Oncol Biol Phys. 1995;31(3):453-9. doi: 10.1016/0360-3016(95)93160-9. [PubMed: 7852106].

6. Crossen JR, Garwood D, Glatstein E, Neuwelt EA. Neurobehavioral sequelae of cranial irradiation in adults: a review of radiationinduced encephalopathy. J Clin Oncol. 1994;12(3):627-42. doi: 10.1200/JCO.1994.12.3.627. [PubMed: 8120563].

7. Roman DD, Sperduto PW. Neuropsychological effects of cranial radiation: current knowledge and future directions. Int J Radiat Oncol Biol 
Phys. 1995;31(4):983-98. doi: 10.1016/0360-3016(94)00550-8. [PubMed: 7860415].

8. Vigliani MC, Sichez N, Poisson M, Delattre JY. A prospective study of cognitive functions following conventional radiotherapy for supratentorial gliomas in young adults: 4-year results. Int J Radiat Oncol Biol Phys. 1996;35(3):527-33. doi: 10.1016/S0360-3016(96)80015-0. [PubMed: 8655376].

9. Ris MD, Noll RB. Long-term neurobehavioral outcome in pediatric brain-tumor patients: review and methodological critique. J Clin Exp Neuropsychol. 1994;16(1):21-42. doi: 10.1080/01688639408402615. [PubMed: 8150888].

10. Stehbens JA, Kaleita TA, Noll RB, MacLean WJ, O’Brien RT, Waskerwitz MJ, et al. CNS prophylaxis of childhood leukemia: what are the longterm neurological, neuropsychological, and behavioral effects? Neuropsychol Rev.1991;2(2):147-77. [PubMed: 1844707].

11. Archibald YM, Lunn D, Ruttan LA, Macdonald DR, Del Maestro RF, Barr $\mathrm{HW}$, et al. Cognitive functioning in long-term survivors of high-grade glioma. J Neurosurg. 1994;80(2):247-53. doi: 10.3171/jns.1994.80.2.0247. [PubMed: 8283263].

12. Hochberg FH, Slotnick B. Neuropsychologic impairment in astrocytoma survivors. Neurology. 1980;30(2):172-7. doi: 10.1212/WNL.30.2.172. [PubMed: 6243762].

13. Imperato JP, Paleologos NA, Vick NA. Effects of treatment on long-term survivors with malignant astrocytomas. Ann Neurol. 1990;28(6):818-22. doi:10.1002/ana.410280614. [PubMed: 2178330].

14. Maire JP, Coudin B, Guerin J, Caudry M. Neuropsychologic impairment in adults with brain tumors. Am J Clin Oncol. 1987;10(2):156-62. doi:10.1097/00000421-198704000-00052. [PubMed: 3565314].

15. Choucair AK, Scott C, Urtasun R, Nelson D, Mousas B, Curran W. Quality of life and neuropsychological evaluation for patients with malignant astrocytomas: RTOG 91-14. Radiation Therapy Oncology Group. Int J Radiat Oncol Biol Phys. 1997;38(1):9-20. doi: 10.1016/S03603016(97)00223-X. [PubMed: 9211998].

16. Meyers CA. Neuropsychological deficits in brain tumor patients. Effect of location, chronicity, and treatment. Cancer Bull. 1986;38(30-2).

17. Taphoorn MJ, Schiphorst AK, Snoek FJ, Lindeboom J, Wolbers JG, Karim $\mathrm{AB}$, et al. Cognitive functions and quality of life in patients with lowgrade gliomas: the impact of radiotherapy. Ann Neurol.1994;36(1):4854. doi: 10.1002/ana.410360111. [PubMed: 8024261].

18. Lilja AM, Portin RI, Hamalainen PI, Salminen EK. Short-term effects of radiotherapy on attention and memory performances in patients with brain tumors. Cancer. 2001;91(12):2361-8. [PubMed: 11413526].

19. Weitzner MA, Meyers CA. Cognitive functioning and quality of life in malignant glioma patients: a review of the literature. Psychooncology. 1997;6(3):169-77. doi: 10.1002/(SICI)1099-1611(199709)6:3lt;169::AIDPON269gt;3.0.CO;2-. [PubMed: 9313282].

20. Scoccianti S, Detti B, Cipressi S, Iannalfi A, Franzese C, Biti G. Changes in neurocognitive functioning and quality of life in adult patients with brain tumors treated with radiotherapy. $J$ Neuroon- col. 2012;108(2):291-308. doi: 10.1007/s11060-012-0821-8. [PubMed: 22354791].

21. Talacchi A, Santini B, Savazzi S, Gerosa M. Cognitive effects of tumour and surgical treatment in glioma patients. $J$ Neurooncol. 2011;103(3):541-9. doi: 10.1007/s11060-010-0417-0. [PubMed: 20878206].

22. Thornton AJ, Hegarty TJ, Ten Haken RK, Yanke BR, LaVigne ML, Fraass BA, et al. Three-dimensional treatment planning of astrocytomas: a dosimetric study of cerebral irradiation. Int J Radiat Oncol Biol Phys. 1991;20(6):1309-15. [PubMed: 2045305].

23. Gregor A, Cull A, Traynor E, Stewart M, Lander F, Love S. Neuropsychometric evaluation of long-term survivors of adult brain tumours: relationship with tumour and treatment parameters. Radiother Oncol. 1996;41(1):55-9. doi: 10.1016/S0167-8140(96)91782-X. [PubMed: 8961368].

24. Pouretemad HR, Khatibi A, Ganjavi A, Shams J, Zarei M. Validation of Addenbrooke's cognitive examination (ACE) in a Persianspeaking population. Dement Geriatr Cogn Disord. 2009;28(4):343-7. doi: 10.1159/000252772. [PubMed: 19864908].

25. Habets EJ, Kloet A, Walchenbach R, Vecht CJ, Klein M, Taphoorn MJ. Tumour and surgery effects on cognitive functioning in highgrade glioma patients. Acta Neurochir (Wien). 2014;156(8):1451-9. doi: 10.1007/s00701-014-2115-8. [PubMed: 24879620].

26. Zarghi A, Zali A, Tehrani-Doost M, Seyedfrootan K, Zarrindast M, Seyedfrootan NS. The effect of surgery on cognitive and mental impairments in patients with glioma brain tumor [In Persian]. Iran J Surg. 2012;19(4):16-20.

27. Correa DD, Shi W, Thaler HT, Cheung AM, DeAngelis LM, Abrey LE. Longitudinal cognitive follow-up in low grade gliomas. $J \mathrm{Neu}$ rooncol. 2008;86(3):321-7. doi: 10.1007/s11060-007-9474-4. [PubMed: 17926007].

28. Bosma I, Vos MJ, Heimans JJ, Taphoorn MJ, Aaronson NK, Postma TJ, et al. The course of neurocognitive functioning in high-grade glioma patients. Neuro Oncol. 2007;9(1):53-62. doi: 10.1215/15228517-2006-012. [PubMed: 17018697].

29. Correa DD, DeAngelis LM, Shi W, Thaler HT, Lin M, Abrey LE. Cognitive functions in low-grade gliomas: disease and treatment effects. J Neurooncol. 2007;81(2):175-84. doi: 10.1007/s11060-006-9212-3. [PubMed: 16850104].

30. Laack NN, Brown PD, Ivnik RJ, Furth AF, Ballman KV, Hammack JE, et al. Cognitive function after radiotherapy for supratentorial low-grade glioma: a North Central Cancer Treatment Group prospective study. Int J Radiat Oncol Biol Phys. 2005;63(4):1175-83. doi: 10.1016/j.ijrobp.2005.04.016. [PubMed: 15964709].

31. Prabhu RS, Won M, Shaw EG, Hu C, Brachman DG, Buckner JC, et al. Effect of the addition of chemotherapy to radiotherapy on cognitive function in patients with low-grade glioma: secondary analysis of RTOG 98-02. J Clin Oncol. 2014;32(6):535-41. doi: 10.1200/JCO.2013.53.1830. [PubMed: 24419119]. 